\title{
Expansion Velocities From Different Ions of Planetary Nebulae with [WC]-Type Central Stars
}

\author{
Selene Medina \\ Instituto de Astronomia, UNAM, Apdo. 70-264, México, D.F., 04510, \\ México \\ Miriam Peña \\ Instituto de Astronomia, UNAM, Apdo. 70-264, México, D.F., 04510, \\ México
}

\section{Observations}

High resolution spectroscopic data of a sample consisting of 24 planetary nebulae (PNe) with WR-type nuclei ([WC]) from the list of Tylenda et al. (1993) plus 1 late-[WC] presented by De Marco et al. (2001), and 7 PNe with weak emission line stars (WELS) nuclei listed in Tylenda et al. (1993) are analyzed. The data were collected using the $2.1 \mathrm{~m}$ telescope at the Observatorio Astronómico Nacional, San Pedro Mártir, México and were used to determine the expansion velocity $\left(V_{\text {exp }}\right)$ of the gaseous component in each sample object in the following ions: $[\mathrm{N} \mathrm{II}] \lambda 6584,[\mathrm{O} \mathrm{II}] \lambda 3729,[\mathrm{O}$ III $] \lambda 5007, \mathrm{H} \beta, \mathrm{HeI} \lambda 5876$, and HeII $\lambda 4686$. A sample of 8 non-WR type (typical) PNe were observed as a controlo sample. All observational data were systematically gathered using the REOSC Echelle Spectrograph on 1995 July 29-31, 1996 June 14-17, 1997 August 3-4, 1998 December 11-14, 1999 October 4-7, and 2000 November 1-3. In order to take the highest $V_{\text {exp }}$ of the gesous component in each ion, it was taken the half width of the line at one tenth of the maximum intensity ( $\mathrm{HW} \frac{1}{10} \mathrm{I}$ ) when the line featured either a single Gaussian profile or a Gaussian with extended wings profile. For two-peaks profiles, $V_{\text {exp }}$ was measured taking into account both the peak-to-peak displacement and the HW $\frac{1}{10} \mathrm{I}$ of the lines.

\section{Results}

It was found that the velocity fields from [WC] and typical PNe apparently differs. It is important to be noticed, however, that WELS are rather grouped among the PNe than the [WC]. A tendency could be noticed between the ionization degree $\mathrm{He}^{++} / \mathrm{He}^{+}$and the $V_{\text {exp }}$. There is no object with low $V_{\text {exp }}$ showing a high $\mathrm{He}^{++} / \mathrm{He}^{+}$value. Since $\mathrm{He}^{++} / \mathrm{He}^{+}$is a function of the stellar temperature, this could be reflecting that the gaseous component around stars with stellar temperures $T_{*}>80000 \mathrm{~K}$, should have high $V_{\text {exp. }}$. This tendency applies in all the objects of the sample, regardless the stellar type. In addition, it was found that [WC] objects with low $\mathrm{He}^{++} / \mathrm{He}^{+}$, cover a wider $V_{\text {exp }}$ range than WELS and typical planetary nebulae. This fact may be due to the high mass-loss rates and terminal velocities of the winds in [WC] central stars. 
It was found that objects with electron densities $N_{e}<4000 \mathrm{~cm}^{-3}$, show $V_{\text {exp }}>45 \mathrm{~km} / \mathrm{s}$, while objects with $N_{e}>6000 \mathrm{~cm}^{-3}$ show $V_{\text {exp }}<45 \mathrm{~km} / \mathrm{s}$. Since $N_{e}$ can be considered as an indicator of the age of the nebula, we found that the the $V_{\text {exp }}$ of the nebulae can be seen as an age indicator. Furthermore, it was found that objects with $T_{*}$ between 30000 and $70000 \mathrm{~K}$ have $V_{\text {exp }}$ laying within a range from 30 to $60 \mathrm{~km} / \mathrm{s}$, while objects with $T_{*}$ between 70000 and $200000 \mathrm{~K}$ show $V_{\text {exp }}$ from 40 to $80 \mathrm{~km} / \mathrm{s}$.

\section{Conclusions}

It is evident that typical PNe have a different velocity structure from the [WC]. It is striking to notice that WELS were found to have a $V_{\text {exp }}$ field very much alike to the typical PNe, rather than to the [WC]. This evidence would indicate that WELS do not belong to the [WC] evolutionary track, at least in their nebular behaviour, despite their strong stellar winds. These results suggest that the nebular $V_{\text {exp }}$ increases with the nebular age.

\section{References}

De Marco, O., Crowther, P.A., Barlow, M.J., Clayton, G.C., \& de Koter, A. 2001, MNRAS, 328, 527

Tylenda, R., Acker, A., \& Stenholm, B. 1993, A\&AS, 102, 595

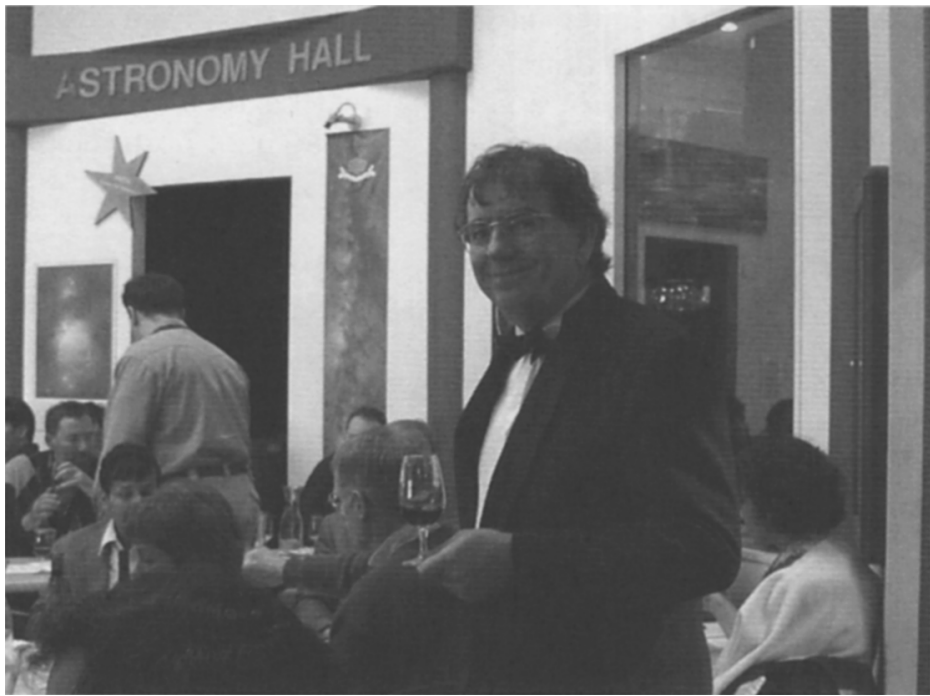

Mike Dopita demonstrating the art of wine tasting. 